\title{
Self Diffusion of Polymers in the Concentrated Regime I. Temperature Dependence of the Self Diffusion Coefficient and the Steady Viscosity of Polystyrene in Dibutyl Phthalate
}

\author{
Norio Nemoto, Takaharu KoJima, Tadashi Inoue, \\ and Michio KURATA \\ Institute for Chemical Research, Kyoto University, \\ Uji, Kyoto 611, Japan
}

(Received May 11, 1988)

\begin{abstract}
The role of the segment friction coefficient in slow chain dynamics in concentrated polymer solutions was studied from temperature dependence of the self diffusion coefficient $D_{\mathrm{s}}$ and the steady viscosity $\eta$. Concentrated solutions of two polystyrene samples (code F4; $\bar{M}_{w}=43900$ and F40; $\bar{M}_{w}=355000$ ) in dibutyl phthalate with concentration of 40 and $50 \mathrm{wt} \%$ were used as test samples. Solutions of their blend containing $1 \mathrm{wt} \%$ of labeled F40 were also studied at total concentration of $40 \mathrm{wt} \%$. For unentangled PS (F4) solutions, temperature dependence of $D_{\mathrm{s}}$ and $\eta$ at each concentration was found to be in good agreement with each other and the functional form was well described in terms of the free volume theory. The function with the same values of the free volume parameters at each concentration is also applicable for description of $T$ dependence of $D_{\mathrm{s}}$ of entangled PS (F40) solutions and also of $D_{\mathrm{s}}$ of F40 in the blend solution. The same segment friction coefficient was concluded to be operative in mass and momentum transports in concentrated solutions irrespective of the entanglement coupling.

KEY WORDS Self Diffusion Coefficient / Steady Viscosity / Concentrated Solution / Polystyrene / Temperature Dependence / Free Volume Theory / Forced Rayleigh Scattering /
\end{abstract}

In recent publications, ${ }^{1,2}$ we postulated that the self diffusion coefficient $D_{\mathrm{s}}$ of flexible polymers in the semidilute regime as well as in melts can be given by the product of two contributions as eq 1 ,

$$
D_{\mathrm{s}}=f_{2}(\zeta) g_{2}(c, M)
$$

Here $f_{2}(\zeta)$ represents contribution from micro Brownian motion of a segment and is a simple function of the segment friction coefficient $\zeta$, i.e., $f_{2} \propto T / \zeta$. The $\zeta$ is supposed, from its definition, to be independent of molecular weight of the polymer and to be a function of concentration $c$ and temperature $T . g_{2}(c, M)$ represents the effect of the topological interac- tion with surrounding polymer chains and depends on $M$ and $c$ in a very complicated manner. ${ }^{3-14}$ Results on temperature dependence of $D_{\text {s }}$ of a polyisoprene melt support eq $1 .{ }^{1}$ In the semidilute regime, data analysis on $D_{\mathrm{s}}$ of polystyrene in tetrahydrofuran ${ }^{2}$ and also recent reports on the translational friction coefficient of poly(methyl methacrylate) in polystyrene solutions ${ }^{15}$ have shown that their behavior can be successfully interpreted by the same idea on which eq 1 is based. Thus a similar study on the concentrated regime seems to be all that remains for establishing the validity of eq 1 over the whole concentration range from the semidilute regime to 
pure melt. By fully specifying dependences of $g_{2}(c, M)$ on the $c$ and $M$, we may get deeper insight on the effects of the topological interaction on slow chain dynamics in condensed systems, which is still under extensive study.

In this paper, we report the results of forced Rayleigh scattering measurements on concentrated solutions of two polystyrene samples and their binary blends in dibutyl phthalate at $c=40$ and $50 \mathrm{wt}^{\circ} \%$ over a wide range of temperature. We also report results of steady viscosity measurements on solutions of the low molecular weight PS. Solutions of the higher molecular weight PS form entanglement networks at these concentrations, but those of the lower molecular weight PS are not entangled. Their temperature dependences are critically compared with one other and are analyzed on the basis of the free volume concept.

\section{EXPERIMENTAL}

\section{Materials}

Two narrow distribution polystyrene samples (code F4; $\bar{M}_{w}=43900$ and F40; $\bar{M}_{w}=$ 355000) were labeled with a photobleachable dye of 2-nitro-4-carboxy-4'-dimethylaminostilbene by following the labelling procedure described in an earlier report. ${ }^{16}$ Concentrated solutions of the labeled homopolymers and their binary blend in dibutyl phthalate (DBP) were prepared for two different concentrations of 40 and $50 \mathrm{wt} \%$ by exactly the same procedure as described earlier. ${ }^{17}$ The solu-
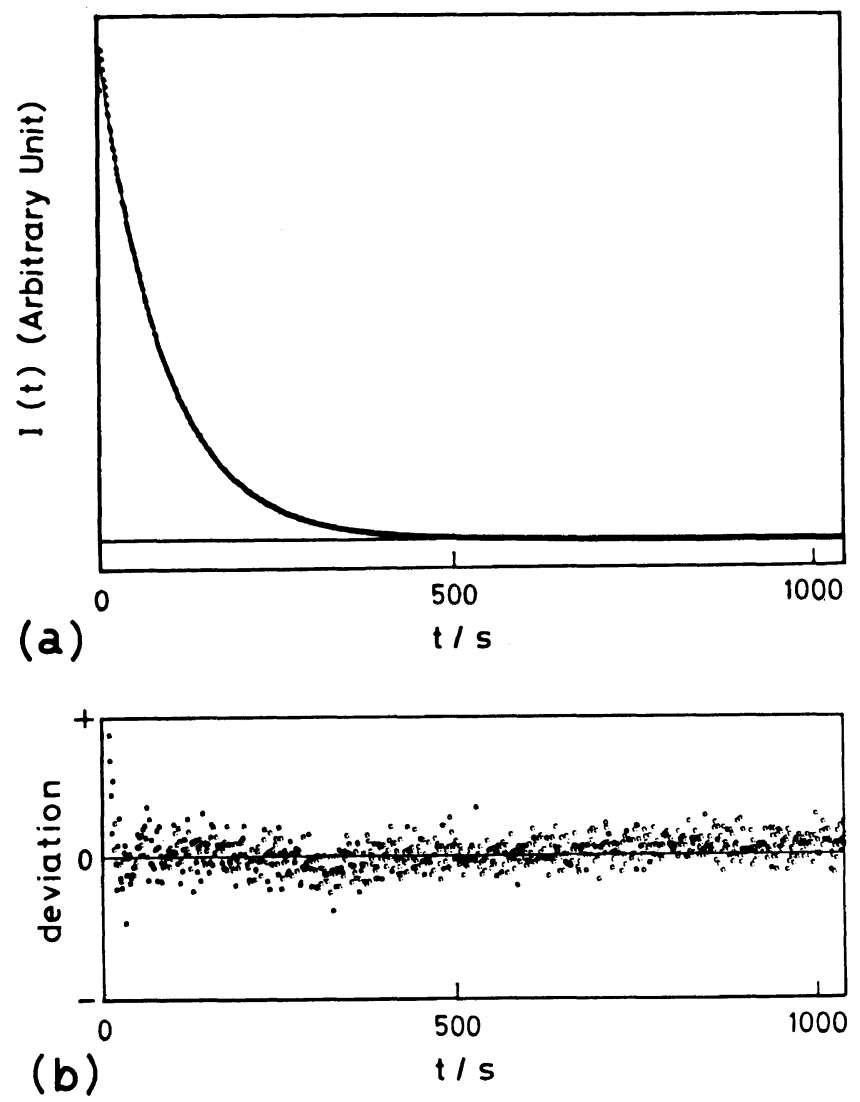

Figure 1. (a) Decay of diffracted light intensity of $I_{\mathrm{d}}$ due to self diffusion of labeled PS with $\bar{M}_{w}=43900$ in DBP at $T=60^{\circ} \mathrm{C}$. Polymer concentration is $40 \mathrm{wt} \%$ and the grating spacing is $8 \mu \mathrm{m}$. The solid curve is a least-square fit with eq 2. (b) Normal residuals obtained from the fit in the upper figure. 
tion of the blend contained $1 \mathrm{wt} \%$ of the labeled higher molecular weight $\mathrm{PS}(\mathrm{F} 40)$ and $39 \%$ of the unlabeled lower molecular weight PS(F4).

\section{Methods}

Forced Rayleigh scattering measurements were performed with an instrument recently constructed at our laboratory. ${ }^{16}$ Diffracted light intensity $I_{\mathrm{d}}$ at the Bragg angle from the solutions was measured as a function of time over a temperature range from 25 to $120^{\circ} \mathrm{C}$ under temperature control of $\pm 0.1^{\circ} \mathrm{C}$. A typical example of raw data is shown in Figure 1. The time profile of $I_{\mathrm{d}}$ could be fitted by eq 2 with a single decay rate $\Gamma_{\mathrm{d}}$ to all data.

$$
I_{\mathrm{d}}=\left\{A \exp \left(-\Gamma_{\mathrm{d}} t\right)+B\right\}^{2}+C^{2}
$$

Here $A$ is amplitude, $B$ and $C$ are contributions from coherent and incoherent background optical field, respectively. The $\Gamma_{\mathrm{d}}$ was estimated from a least-square fit to an accuracy of $10 \%$ at all temperatures. The self diffusion coefficient $D_{\mathrm{s}}$ of the polymers in solutions was calculated as the slope in the linear plot of $\Gamma_{\mathrm{d}}$ against square of the wave vector $q$ which is related to the grating spacing

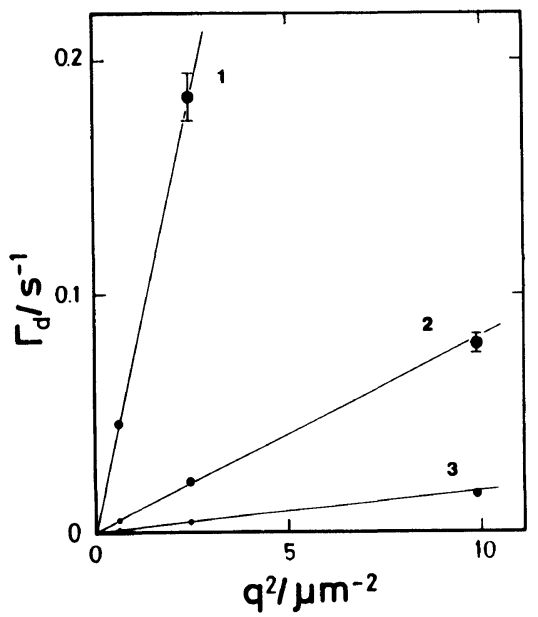

Figure 2. Plot of the decay rate $\Gamma_{\mathrm{d}}$ against square of the wave vector $q$ obtained for the $40 \mathrm{wt} \%$ solution of $\mathrm{F} 4$ in DBP at three temperatures of $1,70^{\circ} ; 2,40^{\circ}$; and 3 , $25^{\circ} \mathrm{C}$. $d$ as $q=2 \pi / d$.

$$
D_{\mathrm{s}}=\Gamma_{\mathrm{d}} / q^{2}
$$

Good linearity, as is shown in Figure 2, assures that $D_{\mathrm{s}}$ was determined to an accuracy of $10 \%$.

The steady viscosity $\eta(\gamma)$ of solutions of the lower molecular weight PS(F4) has been measured as a function of the shear rate $\dot{\gamma}$ with a cone and plate type of viscometer. Details of the viscometer and experimental procedure are described elsewhere. ${ }^{18}$ The zero shear rate value $\eta$ was determined to an accuracy of $10 \%$.

\section{RESULTS AND DISCUSSION}

\section{Temperature Dependence of $D_{\mathrm{s}}$ and $\eta$ of} Unentangled PS-DBP Solutions

In Figure 3, $D_{\mathrm{s}}$ and $\eta$ of the lower molecular weight PS(F4)-DBP solutions at $c=40$ and $50 \mathrm{wt} \%$ are semilogarithmically plotted against the reciprocal of temperature $T^{-1}$. The solutions are not entangled at these concentrations, if the extent of entanglement coupling is estimated by the relation, $M_{\mathrm{e}} c=1.8 \times 10^{4}$ $\left(\mathrm{g} \mathrm{cm}^{-3}\right),{ }^{19}$ where $M_{\mathrm{e}}$ is the molecular weight between entanglements. It is evident from the figure that $T$ dependence of either $D_{\mathrm{s}}$ or $\eta$ can not be represented with the Arrehnius type of equation. The behavior, which is convex to the abscissa for $D_{\mathrm{s}}$ and concave for $\eta$, may be interpreted in terms of the free volume theory as will be shown later.

It is well known that dependences of $\eta$ on $c$, $M$, and $T$ can be discussed with eq 4 in analogy with eq 1 for $D_{\mathrm{s}}{ }^{20}$

$$
\eta=f_{1}(\zeta) g_{1}(c, M)
$$

Here $f_{1}(\zeta)$ is a function of $c$ and $T$, and is supposed to be proportional to $\zeta$, i.e., $f_{1}(\zeta) \propto$ $T / f_{2}(\zeta) \cdot g_{1}(c, M)$ is a function which expresses the effect of the topological interaction on $\eta$. If $g_{1}$ and $g_{2}$ are independent of $T$ to PS solutions as is postulated here, then $T / D_{\mathrm{s}}$ should have the same temperature dependence as $\eta$ does at fixed $c$ and $M$. In Figure 4, reduced quantities $D_{\mathrm{s}, \mathrm{r}} T / D_{\mathrm{s}} T_{\mathrm{r}}$ and $\eta / \eta_{\mathrm{r}}$ are compared. 
N. Nemoto et al.
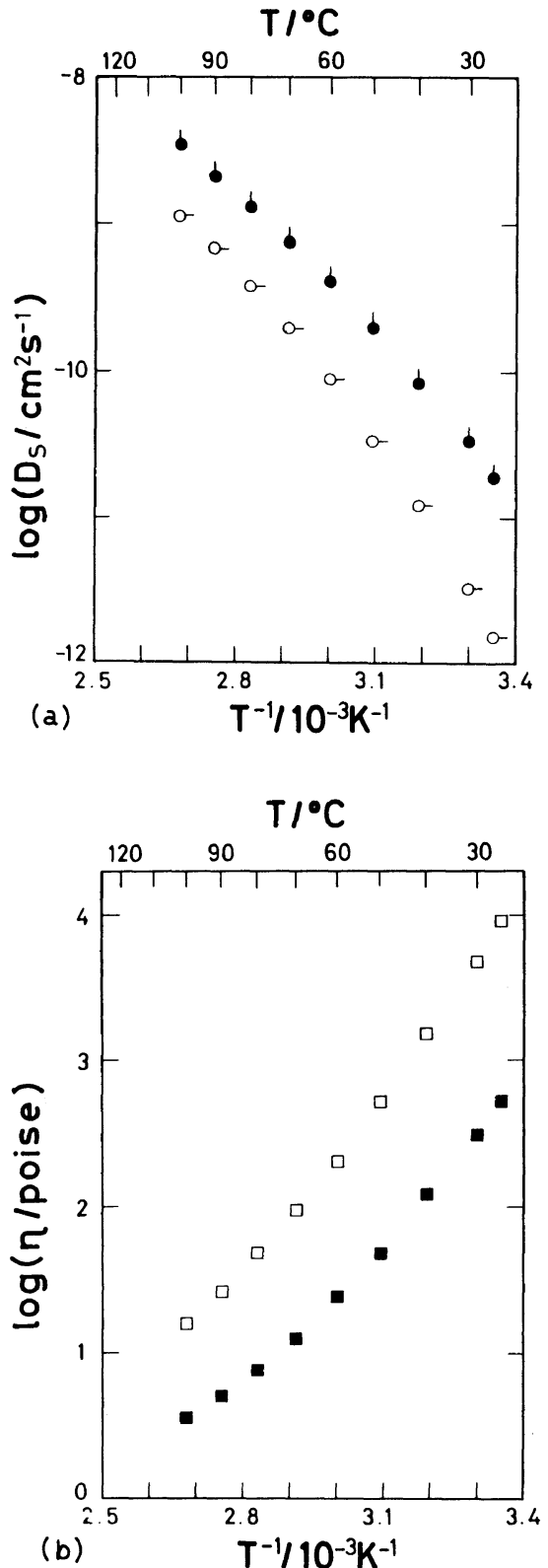

Figure 3. Temperature dependence of the self diffusion coefficient $D_{\mathrm{s}}$ and the steady viscosity $\eta$ of F4 solutions.

(a) Plot of $D_{s}$ vs. $T^{-1}$. Concentration is ( ), 40 and $\left(\mathrm{O}^{-}\right), 50 \mathrm{wt} \%$. (b) Plot of $\eta$ vs. $T^{-1}$. Concentration is

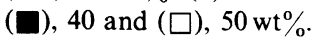

Here $60^{\circ} \mathrm{C}$ is chosen as a reference temperature $T_{\mathrm{r}}$, and $D_{\mathrm{s}, \mathrm{r}}$ and $\eta_{\mathrm{r}}$ are corresponding values at $T_{\mathrm{r}}$. Agreement looks excellent for

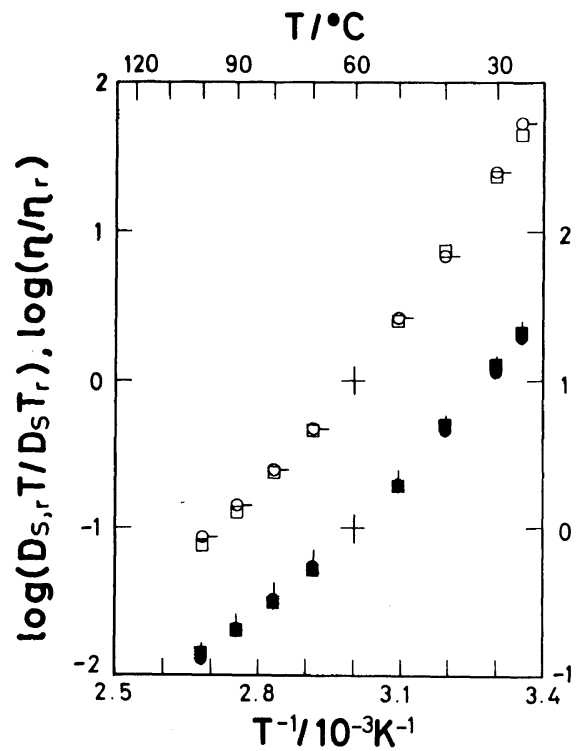

Figure 4. Comparison of temperature dependence between $D_{\mathrm{s}, \mathrm{r}} T / D_{\mathrm{s}} T_{\mathrm{r}}$ and $\eta / \eta_{\mathrm{r}}$ calculated from the data in Figure 3. The reference temperature $T_{\mathrm{r}}$ is $60^{\circ} \mathrm{C}$. Symbols

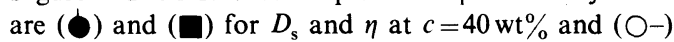
and $(\square)$ for $D_{\mathrm{s}}$ and $\eta$ at $c=50 \mathrm{wt} \%$, respectively.

the reduced data at each concentration. Thus, we can conclude that separation of local and global motions by $f$ and $g$ as is given by eq 1 and 4 is valid for $D_{\mathrm{s}}$ and $\eta$, and that the same segment friction coefficient $\zeta$ may be used to mass and momentum transports at least in unentangled solutions.

Effect of Entanglement Coupling on Temperature Dependence of $D_{\mathrm{s}}$

The higher molecular weight PS sample (F40; $\left.\bar{M}_{w}=355000\right)$ forms an entanglement network at $c=40$ and $50 \mathrm{wt} \%$. Thus, comparison of $D_{s}$ data of these solutions with those of the unentangled PS(F4) solutions in the previous section is quite suitable for examination of the effect of entanglement coupling on $T$ dependence of $D_{\mathrm{s}}$. In addition, we studied $T$ dependence of the tracer diffusion coefficient of F40 $(c=1 \mathrm{wt} \%)$ in the $\mathrm{F} 4$ solution ( $c=$ $39 \mathrm{wt} \%$ ). Values of $D_{\mathrm{s}}$ obtained are tabulated with $D_{\mathrm{s}}$ and $\eta$ values of the F4 solutions in Table I. Figure 5 gives a semilogarithmic plot 
Table I. Self diffusion coefficient $D_{\mathrm{s}}$ and $\eta$ of PS in DBP

\begin{tabular}{|c|c|c|c|c|c|}
\hline $\begin{array}{c}\text { Sample } \\
\text { code }\end{array}$ & $T /{ }^{\circ} \mathrm{C}$ & $\begin{array}{c}D_{\mathrm{s}} \times 10^{11} / \\
\mathrm{cm}^{2} \mathrm{~s}^{-1}\end{array}$ & $\frac{D_{\mathrm{s}, \mathrm{r}} T}{D_{\mathrm{s}} T_{\mathrm{r}}}$ & $\eta /$ poise & $\eta \times \eta_{\mathrm{r}}$ \\
\hline \multirow[t]{9}{*}{ F4 40\% } & 25 & 1.87 & 19.6 & 532 & 21.6 \\
\hline & 30 & 3.30 & 11.3 & 315 & 12.8 \\
\hline & 40 & 8.39 & 4.59 & 123 & 5.00 \\
\hline & 50 & 20.0 & 1.99 & 48.0 & 1.95 \\
\hline & 60 & 41.0 & 1 & 24.6 & 1 \\
\hline & 70 & 76.4 & 0.553 & 12.8 & 0.520 \\
\hline & 80 & 131 & 0.332 & 7.75 & 0.315 \\
\hline & 90 & 212 & 0.221 & 5.10 & 0.207 \\
\hline & 100 & 347 & 0.132 & 3.60 & 0.146 \\
\hline $\mathrm{F} 40$ in $\mathrm{F} 4$ & 25 & 0.292 & 19.3 & & \\
\hline \multirow{7}{*}{$40 \%$} & 30 & 0.513 & 11.2 & & \\
\hline & 40 & 1.27 & 4.66 & & \\
\hline & 50 & 2.92 & 2.09 & & \\
\hline & 60 & 6.30 & 1.00 & & \\
\hline & 70 & 11.4 & 0.569 & & \\
\hline & 80 & 19.4 & 0.344 & & \\
\hline & 90 & 32.4 & 0.212 & & \\
\hline \multirow[t]{8}{*}{$\mathrm{F} 4040 \%$} & 50 & 0.102 & 2.04 & & \\
\hline & 60 & 0.214 & 1.00 & & \\
\hline & 70 & 0.419 & 0.526 & & \\
\hline & 80 & 0.755 & 0.300 & & \\
\hline & 90 & 1.34 & 0.174 & & \\
\hline & 100 & 2.17 & 0.110 & & \\
\hline & 110 & 3.07 & 0.0802 & & \\
\hline & 120 & 3.90 & 0.0648 & & \\
\hline \multirow[t]{9}{*}{$\mathrm{F} 450 \%$} & 25 & 0.152 & 52.7 & 9140 & 44.4 \\
\hline & 30 & 0.327 & 24.9 & 4780 & 23.2 \\
\hline & 40 & 1.22 & 6.90 & 1530 & 7.43 \\
\hline & 50 & 3.31 & 2.62 & 527 & 2.56 \\
\hline & 60 & 8.95 & 1 & 206 & 1 \\
\hline & 70 & 19.7 & 0.468 & 94.0 & 0.456 \\
\hline & 80 & 38.2 & 0.248 & 48.7 & 0.236 \\
\hline & 90 & 68.9 & 0.142 & 26.2 & 0.127 \\
\hline & 100 & 114 & 0.0879 & 16.0 & 0.0777 \\
\hline \multirow[t]{7}{*}{ F40 $50 \%$} & 60 & 0.0382 & 1.00 & & \\
\hline & 70 & 0.0837 & 0.470 & & \\
\hline & 80 & 0.164 & 0.247 & & \\
\hline & 90 & 0.386 & 0.108 & & \\
\hline & 100 & 0.536 & 0.0798 & & \\
\hline & 110 & 0.981 & 0.0448 & & \\
\hline & 120 & 1.51 & 0.0299 & & \\
\hline
\end{tabular}

of $D_{\mathrm{s}}$ vs. $T^{-1}$. The most pronounced feature in the figure is that $D_{\mathrm{s}}$ decreased drastically with the increase in both $c$ and $M$ at constant $T$. If the dependence of $D_{\mathrm{s}}$ on $M$ at constant $c$ and $T$ were boldly expressed by the power law of $D_{\mathrm{s}} \propto M^{-\beta}$, the exponent $\beta$ would become 2.5

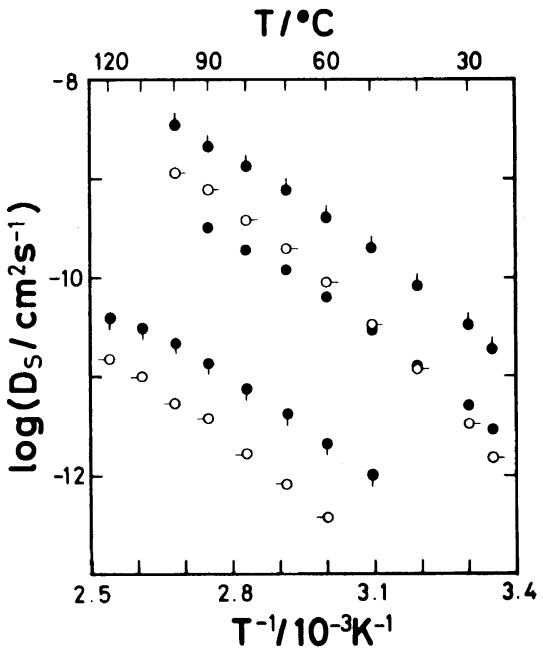

Figure 5. Semilogarithmic plot of $D_{\mathrm{s}} v s . T^{-1}$. Symbols are: (ل), F4, 40\%; (९), F40, 40\%; (O), F40 in F4, 40\%; $(\mathrm{O}-), \mathrm{F} 4,50 \%$; and $(-\mathrm{O}), \mathrm{F} 40,50 \mathrm{wt} \%$.

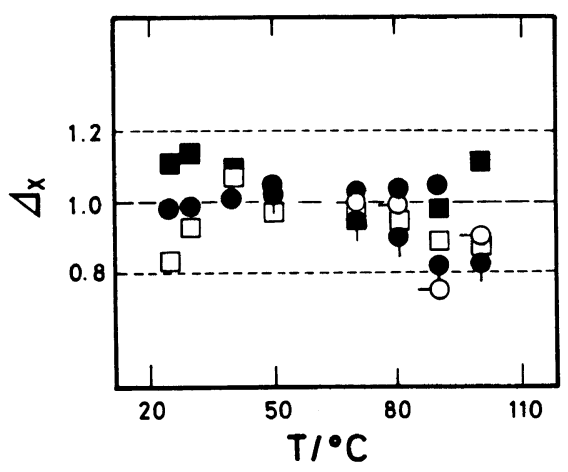

Figure 6. Temperature dependence of $D_{\mathrm{s}}$ and $\eta$ was critically examined by plotting a ratio $\Delta_{x}$, defined by eq 5 , against $T$. Symbols are the same as in Figures 3 and 5 .

and 2.6 at $c=40$ and $50 \mathrm{wt} \%$, respectively. Such a strong $M$ dependence in the transition region from the unentangled to entangled state is consistent with the self diffusion behavior of the same PS molecule in melts around $M / M_{\mathrm{e}} \simeq 1$ where the value $\beta$ has been found to be close to $2.5^{21,22}$ On the other hand, decrease in $D_{\mathrm{s}}$ with increasing $c$ may be mainly related to increase in local friction. More detailed studies on dependences of $D_{\mathrm{s}}$ on $c$ and $M$ will be reported in forthcoming papers.

In spite of the strong dependence of $D_{\mathrm{s}}$ on $c$ 
and $M$, relative changes in $D_{\mathrm{s}}$ values with varying temperature look almost the same for two homopolymer solutions and one blend solution with the same total concentration. For a quantitative comparison, a reduced quantity $a_{x}=\left[D_{\mathrm{s}, \mathrm{r}} T / D_{\mathrm{s}} T_{\mathrm{r}}\right]_{x}$ was first calculated from $D_{\mathrm{s}}$ data in Figure 5 by taking the reference temperature $T_{\mathrm{r}}$ as $60^{\circ} \mathrm{C}$. Here the suffix $x$ denotes the $\mathrm{F} 40$ or $\mathrm{F} 4$ solutions, or the blend solution. Then a ratio $\Delta_{x}$, defined by eq 5 , has been calculated and is plotted against $T$ in Figure 6.

$$
\Delta_{x}=a_{x} / a_{\mathrm{F} 4}
$$

In the figure, we also show $\Delta_{x}$ of $\eta$ of the F4 solutions estimated by the same procedure. The $\Delta_{x}=1$ in the ordinate means that the temperature dependence of $D_{\mathrm{s}}$ or $\eta$ of the solution denoted by the suffix $x$ is identical with that of $D_{\mathrm{s}}$ of the F4 solution at the corresponding concentration. If there is a $10 \%$ difference between $a_{x}(T)$ and $a_{\mathrm{F} 4}(T)$, it results in $\Delta_{x}=1.1$ or 0.9 . All $\Delta_{x}$ values are located in the range from 0.8 to 1.2 except only one $\Delta_{x}$ value for $D_{\mathrm{s}}$ of the $50 \%$ F40 solution at $90^{\circ} \mathrm{C}$. In consideration of an experimental accuracy of $10 \%$ for both quantities of $D_{\mathrm{s}}$ and $\eta$, agreement seems satisfactory. Thus the temperature dependence of $D_{\mathrm{s}}$ is unaffected by the presence of entanglement coupling. The above results may be regarded as strong support of the applicability of eq 1 to $D_{\mathrm{s}}$ over a wide concentration range from the semidilute region to pure polymer melts.

\section{Analysis of the Data in Terms of the Free Volume Theory}

It was pointed out in a previous section that $T$ dependence of $D_{\mathrm{s}}$ and $\eta$ (Figures 3 and 5) did not obey the simple Arrehnius type of equation. We here apply the free volume theory for interpretation of the data, because the theory has been demonstrated to be quite successful for explaining the $T$ dependence of viscoelastic properties of polymeric materials in concentrated solutions and in bulk. ${ }^{23}$ If $D_{\mathrm{s}}$ and $\eta$ can be expressed by the product of the local friction factor $f$ and the global structure factor $g$ as given by eq 1 and 4 , reduced quantities such as $D_{\mathrm{s}, \mathrm{r}} T / D_{\mathrm{s}} T_{\mathrm{r}}$ and $\eta / \eta_{\mathrm{r}}$ are equal to a ratio of the segment friction coefficient $\zeta$ at $T$ and $T_{\mathrm{r}}$. Their temperature dependences may be expressed in terms of the WLF type of equation,

$$
\begin{aligned}
\log \left(D_{\mathrm{s}, \mathrm{r}} T / D_{\mathrm{s}} T_{\mathrm{r}}\right) & =\log \left(\eta / \eta_{\mathrm{r}}\right) \\
& =\log \left(\zeta(T) / \zeta\left(T_{\mathrm{r}}\right)\right) \\
& =-\frac{C_{1}{ }^{\mathrm{r}}\left(T-T_{\mathrm{r}}\right)}{C_{2}{ }^{\mathrm{r}}+\left(T-T_{\mathrm{r}}\right)}
\end{aligned}
$$

Here $C_{1}{ }^{\mathrm{r}}$ and $C_{2}{ }^{\mathrm{r}}$ are constants and related to the free volume parameters, the fractional free volume, $f_{\mathrm{r}}$, and the thermal expansion coefficient of the free volume, $\alpha_{\mathrm{f}}$, by eq 7 .

$$
C_{1}{ }^{\mathrm{r}}=B / 2.303 f_{\mathrm{r}}, \quad C_{2}{ }^{\mathrm{r}}=f_{\mathrm{r}} / \alpha_{\mathrm{f}}
$$

where $B$ is a constant of an order of unity. Equation 6 implies that a plot of $\left(T-T_{\mathrm{r}}\right) /$ $\log \left(\zeta(T) / \zeta\left(T_{\mathrm{r}}\right)\right)$ against $T-T_{\mathrm{r}}$ should give a

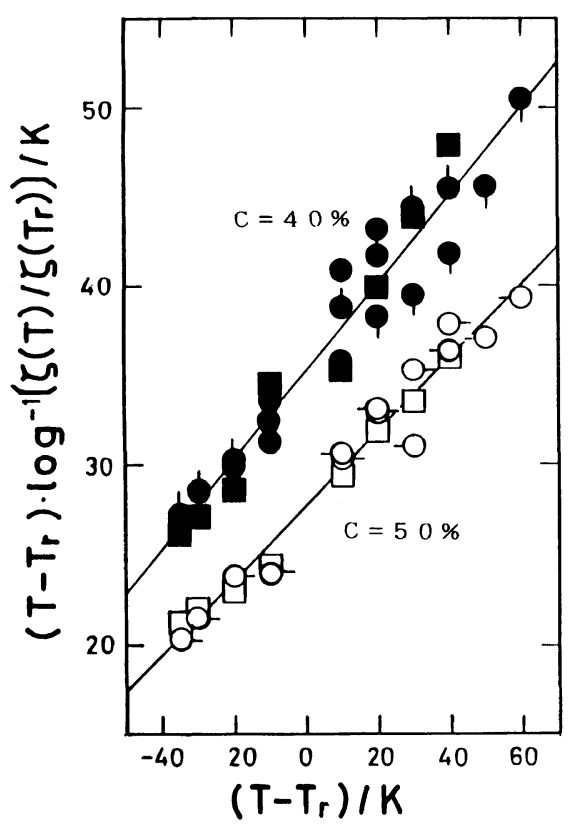

Figure 7. Applicability of WLF equation to temperature dependence of $D_{\mathrm{s}}$ and $\eta$ in Figures 3 and 5 was tested. Symbols are the same as in Figures 3 and 5. Solid lines are the best fit from eq 6 . 
Table II. Free volume parameters

\begin{tabular}{ccccc}
\hline$c$ & & $C_{2}{ }^{\mathrm{r}}$ & & $\alpha_{\mathrm{f}}$ \\
\hline $\mathrm{wt} \%$ & $C_{1}{ }^{\mathrm{r}}$ & $\frac{\mathrm{K}}{\mathrm{r}}$ & & $\frac{10^{-4} \mathrm{~K}^{-1}}{40}$ \\
\hline 50 & 4.06 & 143. & 0.107 & 7.49 \\
\hline
\end{tabular}

straight line. When the $D_{\mathrm{s}}$ and $\eta$ data are plotted in such a manner, we obtain a straight line at each $c$, as is shown in Figure 7 , from which $C_{1}{ }^{\mathrm{r}}$ and $C_{2}{ }^{\mathrm{r}}$ are easily evaluated. The $\alpha_{\mathrm{f}}$ and $f_{\mathrm{r}}$ were calculated from eq 7 by putting $B=1$, and are listed with values of $C_{1}{ }^{r}$ and $C_{2}{ }^{r}$ in Table II. The thermal expansion cofficient of DBP and PS above $T_{\mathrm{g}}$ is $8.6 \times 10^{-4}$ and $6.5 \times 10^{-4} \mathrm{~K}^{-1}$, respectively. ${ }^{24}$ The $\alpha_{\mathrm{f}}$ values of the two PS-DBP solutions in Table II are intermediate between them. The glass transition temperature $T_{\mathrm{g}}$ of the solutions was roughly estimated from DSC measurements as $-55^{\circ}$ for $c=40 \mathrm{wt} \%$ and $-38^{\circ} \mathrm{C}$ for $50 \mathrm{wt} \%$, respectively. Assuming linear dependence of $f$ on $T$, the fractional free volume at $T_{\mathrm{g}}$ was calculated as 0.021 and 0.024 for the solutions with $c=40$ and $50 \mathrm{wt} \%$, respectively. The values are in reasonable agreement with the accepted value of $f_{\mathrm{g}}=0.025 \pm 0.003$. $^{23}$

Acknowledgements. We are very grateful to Mr. T. Yamagishi of this institute for DSC measurements. We also thank to Toyo Soda Co., Ltd. for supplies of PS samples. This work was partly supported by a Grant-in-Aid for Scientific Research (No. 62550653) of Ministry of Culture, Science and Education of Japan.

\section{REFERENCES}

1. N. Nemoto, M. R. Landry, I. Noh, and H. Yu, Polym. Commun., 25, 141 (1984).
2. N. Nemoto, M. R. Landry, I. Noh, T. Kitano, J. A. Wesson, and H. Yu, Macromolecules, 18, 308 (1985).

3. L. Leger, H. Hervet, and F. Rondelez, Macromolecules, 14, 1732 (1981).

4. P. T. Callaghan and D. N. Pinder, Macromolecules, 17, 431 (1984).

5. J. A. Wesson, I. Noh, T. Kitano, and H. Yu, Macromolecules, 17, 782 (1984).

6. H. Kim, T. Chang, J. H. Yohanan, L. Wang, and H. Yu, Macromolecules, 19, 2737 (1986).

7. H. Deschamps and L. Leger, Macromolecules, 19, 2760 (1986).

8. C. R. Bartles, B. Christ, and W. W. Graessley, Macromolecules, 17, 2702 (1984).

9. (a) M. Antonietti, J. Coutandin, R. Grutter, and H. Sillescu, Macromolecules, 17, 798 (1984).

(b) M. Antonietti, J. Coutandin, and H. Sillescu, Macromolecules, 19, 793 (1986).

10. (a) B. A. Smith, Macromolecules, 15, 469 (1982).

(b) B. A. Smith, E. T. Samulski, L.-P. Yu, and M. A Winnik, Macromolecules, 18, 1901 (1985).

(c) B. A. Smith, S. J. Mumby, E. T. Samulski, and L.P. Yu, Macromolecules, 19, 470 (1986).

11. (a) P. F. Green, P. J. Mills, C. J. Palmstron, J. W. Mayer, and E. J. Kramer, Phys. Rev. Lett., 58, 2145 (1984).

(b) P. F. Green and E. J. Kramer, Macromolecules, 19, 1108 (1986).

12. G. Fleisher, Polym. Bull., 11, 75 (1984).

13. D. S. Pearson, G. VerStrate, E. von Meerwall, and F. G. Schilling, Macromolecules, 20, 1133 (1987).

14. Q. Trang-Cong, T. Chang, C. C. Han, and Y. Nishijima, Polymer, 27, 1705 (1986).

15. N. Nemoto, S. Okada, T. Inoue, and M. Kurata, Macromolecules, 21, 1502 (1988).

16. T. Inoue, N. Nemoto, T. Kojima, Y. Tsunashima, and M. Kurata, Nihon Reorogi Gakkaishi, 16, 72 (1988) (in Japanese).

17. T. Inoue, N. Nemoto, T. Kojima, and M. Kurata, the preceeding paper.

18. N. Nemoto, K. Okawa, and H. Odani, Bull. Inst. Chem. Res., Kyoto Univ., 51, 118 (1973).

19. K. Osaki, K. Nishizawa, and M. Kurata, Macromolecules, 15, 1068 (1982).

20. G. C. Berry and T. G. Fox, Adv. Polym. Sci., 5, 261 (1968).

21. H. Watanabe and T. Kotaka, Macromolecules, 20, 530 (1987).

22. N. Nemoto, S. Okada, T. Inoue, and M. Kurata, Macromolecules, 21, 1509 (1988).

23. J. D. Ferry, "Viscoelastic Properties of Polymers," 3rd ed, John Wiley, New York, N. Y., 1980.

24. J. A. Riddick and W. B. Bunger, "Organic Solvents," 3rd ed, John Wiley, New York, N. Y., 1980. 